\title{
Fecal Microbiota Transplantation: Current Applications, Effectiveness, and Future Perspectives
}

\author{
Hyun Ho Choi and Young-Seok Cho \\ Department of Internal Medicine, Seoul St. Mary's Hospital, College of Medicine, The Catholic University of Korea, Seoul, Korea
}

Fecal microbiota transplantation (FMT) is the infusion of liquid filtrate feces from a healthy donor into the gut of a recipient to cure a specific disease. A fecal suspension can be administered by nasogastric or nasoduodenal tube, colonoscope, enema, or capsule. The high success rate and safety in the short term reported for recurrent Clostridium difficile infection has elevated FMT as an emerging treatment for a wide range of disorders, including Parkinson's disease, fibromyalgia, chronic fatigue syndrome, myoclonus dystopia, multiple sclerosis, obesity, insulin resistance, metabolic syndrome, and autism. There are many unanswered questions regarding FMT, including donor selection and screening, standardized protocols, long-term safety, and regulatory issues. This article reviews the efficacy and safety of FMT used in treating a variety of diseases, methodology, criteria for donor selection and screening, and various concerns regarding FMT. Clin Endosc 2016;49:257-265

Key Words: Fecal microbiota transplantation; Clostridium difficile infection; Colitis, ulcerative; Crohn disease; Irritable bowel syndrome

\section{INTRODUCTION}

The gut microbiota provides an intestinal biological barrier against pathogens and has a pivotal role in the maintenance of intestinal homeostasis and modulation of the host immune system. ${ }^{1}$ The specific changes in the composition of gut microbiota, termed dysbiosis, have been associated not only with many gastrointestinal (GI) diseases but also with metabolic diseases, autoimmune diseases, allergic disorders, and neuropsychiatric disorders. ${ }^{2}$ Restoring a healthy microbial community is therefore a promising therapeutic strategy for diseases related with gut dysbiosis. ${ }^{3}$ Fecal microbiota trans-

Received: September 17, 2015 Revised: October 26, 2015

Accepted: October 27, 2015

Correspondence: Young-Seok Cho

Division of Gastroenterology, Department of Internal Medicine, Seoul St. Mary's Hospital, College of Medicine, The Catholic University of Korea, 222 Banpo-daero, Seocho-gu, Seoul 06591, Korea

Tel: +82-2-2258-6021, Fax: +82-2-2258-2038, E-mail: yscho@catholic.ac.kr

(cc) This is an Open Access article distributed under the terms of the Creative Commons Attribution Non-Commercial License (http://creativecommons.org/ licenses/by-nc/3.0) which permits unrestricted non-commercial use, distribution, and reproduction in any medium, provided the original work is properly cited. plantation (FMT), also called stool/fecal transplantation or fecal bacteriotherapy, is the infusion or engraftment of liquid filtrate feces from a healthy donor into the gut of a recipient to cure a specific disease. ${ }^{4}$ The concept of FMT for treatment of human GI disease was described approximately 1,700 years ago by a Chinese medical scientist named Ge Hong. ${ }^{5}$ At that time, he orally administered human fecal suspension to treat patients who had food poisoning or severe diarrhea. Borody et al. ${ }^{4}$ and Brandt et al. ${ }^{6}$ noted that FMT may have been first used in veterinary medicine by the Italian anatomist Fabricius Aquapendente in the 17th century. It was first reported in the English language by Eiseman et al., ${ }^{7}$ who used fecal enemas to treat pseudomembranous colitis in 1958. Recently, FMT is becoming interesting with its effectiveness in treating refractory and recurrent Clostridium difficile infection (CDI) and the possibilities for treating other diverse conditions. ${ }^{8} \mathrm{We}$ review the efficacy of FMT used in treating a variety of diseases and preclinical conditions. In addition, we describe the methodology, criteria for donor selection and screening, and safety data. 


\section{PATHOPHYSIOLOGIC RATIONALE FOR FMT}

The human gut microbiota is complex community of microorganisms, which include 100 trillion $\left(10^{14}\right)$ bacteria, quadrillion viruses, fungi, parasites, and archaea. ${ }^{1}$ The "normal" gut microbiota consists of 500 to 1,000 species that belong to only a few bacterial phyla. ${ }^{9,10}$ The most abundant bacteria in the human gut are the Bacteroidetes and Firmicutes phyla, but other bacterial species mostly belong to members of the phyla Actinobacteria, Fusobacteria, Proteobacteria, Verrucomicrobia, and Cyanobacteria. ${ }^{9,10}$ Microbial colonization of the human gut begins during birth. Each individual has his or her own specific gut microbiota, of which the composition is influenced by various environmental factors, including diet, lifestyle, the use of antibiotics and hygiene preferences. ${ }^{11}$ It is essential for several aspects of host biology, including the metabolism of indigestible polysaccharides, production of essential vitamins, development and differentiation of the host's intestinal epithelium and immune system, maintenance of tissue homeostasis, and protection against the invasion of pathogens. ${ }^{11}$

Gut dysbiosis is associated with various diseases, including CDI, inflammatory bowel disease (IBD), irritable bowel syndrome (IBS), metabolic syndrome, obesity, types 1 and 2 diabetes, atopy, multiple sclerosis, autism, colorectal cancer, etc. ${ }^{3}$ The protective effect of normal gut flora on CDI is well recognized. ${ }^{12}$ Among several factors causing gut dysbiosis, the use of drugs, particularly antibiotics, is the most important inciting factor. ${ }^{13}$ Antibiotics affect both the overall size of the gut bacterial community and the composition of the community, producing an environment that allows germination of $C$. difficile spores and expansion of the pathogen. ${ }^{12}$ FMT restores gut microbiota diversity via the infusion of donor feces into the GI tract of a patient with CDI. Khoruts et al. ${ }^{14}$ used molecular approaches to characterize the bacterial composition of the colonic microbiota before and after FMT in a patient with CDI. Before FMT, the patient's residual colonic microbiota was deficient in members of the normally dominant phyla, Bacteroidetes and Firmicutes. By 2 weeks after FMT, the recipient's fecal bacteria composition resembled the donor's and was dominated by Bacteroides spp. strains and an uncharacterized butyrate-producing bacterium. These changes were accompanied by symptom resolution and were long lasting. The suggested mechanisms are following: (1) prevention of colonization by toxigenic $C$. difficile through competition for a limited amount of nutrients; (2) direct inhibition of $C$. difficile growth and its toxigenic activity; (3) modulation of metabolites and transformation of bile acids, which indirectly impairs the life cycle of $C$. difficile; (4) physiologic cross talk between the normal flora and the host immune system, resulting in a regulated immune response that may prevent colonization by C. difficile and its recurrence. ${ }^{15}$

Although no definitive pathogen has been detected as the etiologic factor of IBD and a definite infectious cause cannot be considered alone, many studies have investigated a disturbance of the intestinal microbiota as a contributing factor to the pathogenesis of IBD. ${ }^{16}$ Gut dysbiosis in IBD is characterized by a decreased diversity at the species level, with decreases in the Bacteroides phylum and the Lachnospiraceae group within the Firmicutes phylum and a relative increase in proinflammatory bacteria such as Proteobacteria and Actinobacteria. ${ }^{17,18}$ In addition, a decrease in a butyrate-producing bacterium, which is important in intestinal health, has been observed in patients with IBD. ${ }^{16,19}$ In IBD, abnormal microbial colonization of the GI tract may be the origin of excessive or dysregulated immune response, resulting in chronic inflammation and the development of mucosal lesions. ${ }^{18}$ However, it is still unclear whether dysbiosis itself causes IBD or if it represents an epiphenomenon due to microbial alterations as a consequence of the disease. ${ }^{19}$ Several studies reported that FMT may be a promising approach for the management of diseases associated with gut dysbiosis, including IBD. ${ }^{20}$

\section{CLINICAL APPLICATIONS}

\section{C. difficile infection}

In 1978, C. difficile was first identified as the cause of pseudomembranous colitis. ${ }^{21}$ Because of high rate of recurrence (15\% to $30 \%$ ), patients with CDI have higher health-care burden. ${ }^{8}$ In the past few decades, FMT has received considerable attention because of a convincing clinical trial of treatment of recurrent CDI. The first randomized controlled trial (RCT) of FMT for 43 patients with recurrent CDI compared FMT administered via nasoduodenal tube after 4 to 5 days of oral vancomycin with 14 days of continued vancomycin alone and with 14 days of vancomycin plus bowel lavage. ${ }^{22}$ Symptoms resolved within 3 months in $81 \%$ of patients receiving FMT, in $31 \%$ of those receiving vancomycin alone, and in $23 \%$ of patients receiving vancomycin plus bowel lavage. The study was terminated early because FMT was more than twice as effective in resolving symptoms as antibiotics alone. The second RCT compared two FMT treatment approaches, via nasogastric tube and via colonoscopy, in 20 patients. ${ }^{23}$ Symptoms resolved completely for $70 \%$ of patients after single FMT, and the overall cure rate was $90 \%$ after retreatment; the difference between treatment approaches was not significant $(60 \%$ in the nasogastric tube group and $80 \%$ in the colonoscopy group; $p=0.63$ ). However, this study was 
unblinded and did not include a non-FMT control group. The first systematic review published in 2011 included 317 patients with recurrent CDI treated with FMT across 27 cases and reports. ${ }^{24}$ Symptoms resolved in $92 \%$ of patients, $89 \%$ after a single treatment, and 5\% after retreatment due to failure or relapse. A recent systematic review published in 2015 included two RCTs, 28 case-series studies, and five case reports. ${ }^{8}$ The results showed that FMT was successful in $85 \%$ of recurrent CDI and $55 \%$ of refractory CDI compared with $30 \%$ to $80 \%$ success rates for medical therapies. Although FMT has a substantial effect and few short-term side effects for adults with recurrent CDI, there is insufficient evidence regarding FMT for patients with refractory $\mathrm{CDI}$ or for initial treatment of CDI. Considerations for future study in CDI include the need for a large, blinded RCT that would compare FMT with placebo in patients randomized after standard antimicrobial therapies, the best source and processing methods for donor stool, and the best timing for FMT after antimicrobial use. The two most recent guidelines differ about the strength of evidence supporting FMT-a European guideline stated that FMT is "strongly recommended (A-I)" after a second recurrence of $\mathrm{CDI},{ }^{25}$ whereas a guideline from the American College of Gastroenterology offered a more cautious recommendation, stating that "if there is a third recurrence after a pulsed vancomycin regimen, FMT should be considered (conditional recommendation, moderate quality evidence). ${ }^{326}$ FMT for CDI is a topic of considerable research. Despite the high success rate of FMT in treating recurrence, its wider practice is hindered by several factors, including concerns about pathogen transmission, limited viability of fresh samples, lack of reimbursement for donor screening, difficulty in stool preparation and administration, concerns about doing the procedure in the endoscopy laboratory or medical office due to odor, difficulty in convincing patients, and lack of standardized treatment regimen. ${ }^{23,27-29}$ Patel et al. ${ }^{30}$ reported a successful outcome in two patients with recurrent CDI unresponsive to repeated courses of antibiotics who received a stool substitute, a preparation of 33 different intestinal bacteria isolated in pure culture from a single donor. Recent studies demonstrated that FMT using a frozen inoculum from carefully screened health volunteer donor is effective for treating recurrent CDI. ${ }^{23,31}$ The outcomes of this approach was similar with those of FMT with fresh stools, suggesting that preparation of frozen transplants can simplify the practical aspects of FMT without loss of efficacy or safety. ${ }^{29}$ A more recent feasibility study used frozen fecal capsules, prepared from prescreened healthy donor for treating 20 patients with recurrent $\mathrm{CDI}{ }^{28}$ The results showed an overall $90 \%$ rate of clinical resolution of diarrhea after one or two treatment courses. In Korea, Gweon et al. ${ }^{32}$ first reported two cases of refractory pseudomembranous colitis treated with FMT in 2013. Subsequent case reports showed FMT can cure $\mathrm{CDI}$ complicated by acute respiratory distress syndrome, ${ }^{33}$ toxic megacolon by $\mathrm{CDI},{ }^{34}$ and $\mathrm{CDI}$ in a patient colonized by vancomycin-resistant enterococcus. ${ }^{35}$

\section{Inflammatory bowel disease}

FMT for IBD was first reported in 1989, with one of the authors himself experiencing severely active ulcerative colitis (UC), refractory to aminosalicylates and corticosteroids. ${ }^{36}$ He performed transplantation of a healthy donor stool by retention enema. Symptoms disappeared for 6 months after FMT, and active inflammation was not detected at follow-up biopsy sampling of the colon. Another preliminary case report of FMT enema showed significant clinical improvements in several GI conditions including constipation, IBS, UC, and Crohn disease $(\mathrm{CD}){ }^{37}$ These studies were followed by anecdotal reports and small case series in patients with IBD and combined CDI, showing a beneficial effect of FMT. ${ }^{4,38,39}$ The first systematic review was published in 2012 and included 41 patients with IBD (27 UC, $12 \mathrm{CD}$, and two unclassified) treated with FMT via enema, colonoscopy, or nasojejunal tube. ${ }^{40}$ Among all the patients, 15 patients received FMT for treatment of combined CDI and 26 patients received FMT for treatment of IBD. The majority of the patients for IBD treatment experienced a reduction of symptoms (19/25), cessation of IBD medications (13/17), and endoscopic and histologic disease remission (15/24). However, the high rates (63\%) of remission in this review may be due to publication bias because the study did not include a complete clinical overview of patients achieving disease remission. This review was limited to the nine small case series/reports available at the time. A second systematic review of 111 patients reported a "success rate" of $77.8 \%$ for adult IBD patients. ${ }^{41}$ However, this study also had several methodological limitations; notably, the clinical outcomes after FMT were assessed by treatment success rate and not by using any other, more validated measures. A recent systematic review and meta-analysis analyzed 18 studies (nine cohort studies, eight case studies, and one RCT) on FMT that included 122 patients with IBD (79 UC, $39 \mathrm{CD}$, and four unclassified) ${ }^{20}$ In this study, clinical remission after FMT was achieved in 54 (45\%) of 119 patients. However, the pooled estimate for achieving clinical remission was $36.2 \%$, when the case series were excluded to minimize publication bias. In subgroup analyses, the pooled estimates were $22 \%$ in UC, $60.5 \%$ in CD, and $64.1 \%$ in young patients (age 7 to 20 years), respectively. Although it might appear that FMT is more effective in $\mathrm{CD}$ or younger patients, the patient populations were significantly heterogeneous, limiting the applicability of these conclusions. 
Recently, the first RCTs evaluating the efficacy of FMT in UC were reported. ${ }^{42,43}$ In the study reported by Moayyedi et al., ${ }^{42} 75$ patients with mild to moderate UC were randomized to weekly FMT or placebo (water) via retention enema for 6 weeks. The primary end point was remission of UC, defined as a Mayo score of $<3$ with complete mucosal healing at week 7. Remission was achieved in $24 \%$ of patients receiving FMT and $5 \%$ with placebo, and the difference in remission was statistically significant. In addition, stool from patients receiving FMT developed an increase in microbial diversity compared with those given placebo. The second study enrolled 50 patients, also with mild to moderate UC, and randomized patients to either donor stool or autologous FMT (infusion of their own stool as placebo) delivered via nasoduodenal tube at baseline and again 3 weeks later. ${ }^{43}$ The primary end point was clinical remission combined with $\geq 1$-point decrease in the Mayo score at week 12 . Only 37 patients completed the primary end point assessment. Clinical remission was achieved in $30.4 \%$ of patients receiving FMT and $25 \%$ with autologous FMT, but the difference was not statistically significant. Nevertheless, the microbiota profile of responders of FMT group was similar to that of their respective donors, whereas nonresponders did not show the same trend. Both studies were terminated by their respective data and safety monitoring boards due to futility in reaching primary efficacy point.

Studies investigating the therapeutic efficacy of FMT in CD have been limited to small, uncontrolled series with varying response rates. It has been reported that $\mathrm{CD}$ is less likely to respond to FMT compared with UC. ${ }^{44}$ Although a few case series demonstrated successful outcomes after single infusion, ${ }^{45,46}$ multiple treatment courses are required in the most of the patients with $\mathrm{CD}^{47} \mathrm{~A}$ recent study from China reported the results of single FMT through the mid-gut in 30 patients with refractory $\mathrm{CD}^{48}$ The rates of clinical improvement and clinical remission at the first month were $86.7 \%(26 / 30)$ and $76.7 \%(23 / 30)$, respectively. In addition, the body weights of the patients increased significantly at 3-month assessment point after FMT. A number of RCTs of FMT for IBD, at least four of which are for $\mathrm{CD}$, are registered on clinicaltrials.gov (accessed 2015 Sep 10) and now underway to further characterize treatment efficacy and safety. Taken together, FMT is not nearly as effective in IBD as it is in CDI, suggesting that IBD is a more complicated disease with a complex pathologic interplay among genetic, environmental, immunologic, and gut microbial factors. In future clinical trials, various clinical conditions, such as patients' clinical characteristics, concomitant medications, timing of FMT, and dosage or frequency of FMT, should be considered.

\section{Functional GI disorders}

The current working hypothesis is that abnormal microbiota activates mucosal innate immune responses that increase epithelial permeability, activates nociceptive sensory pathways, and dysregulates the enteric nervous system. ${ }^{49}$ In contrast to IBD, the role of the microbiota in the development of IBS only recently has been considered, but early stage results have been encouraging. The rationale for microbiota-directed interventions for treatment of IBS seems weaker than in IBD, but is emerging. ${ }^{50}$ Although alterations in immunity, motility, and the brain-gut axis have been implicated in disease pathogenesis, the role of the intestinal microbiota are increasing, and numerous studies have demonstrated significant differences from normal in the intestinal flora of functional gastrointestinal disorder (FGID) with those of the healthy population and between types of FGID. ${ }^{51}$ A recent study reported a global and deep molecular analysis of fecal samples from 62 IBS patients and 46 healthy controls and confirmed a statistically significant difference in their intestinal microbiota composition. IBS patients had a significant decrease in the numbers of Bacteroidetes and Bifidobacterium and Faecalibacterium spp. and a significant increase in Firmicutes. ${ }^{52}$ This alteration of intestinal microbiota in IBS patients has been linked to immune dysfunction and altered neurological function such as increased anxiety and decreased pain threshold, suggesting that restoration of normal intestinal homeostasis via FMT may result in symptomatic improvement. ${ }^{51}$ Pinn et al. ${ }^{53}$ reported the efficacy of FMT for the treatment of IBS. They treated 13 patients (nine with IBS with diarrhea, three with IBS with constipation, and one with IBS with a mixed bowel pattern) and observed them for an average of 11 months. Resolution or improvement of symptoms was reported in $70 \%$, including abdominal pain (72\%), bowel habit (69\%), dyspepsia (67\%), bloating (50\%), and flatus (42\%). In a case series of 45 patients with chronic constipation who were treated with FMT via colonoscopy followed by a single fecal enema infusion the next day, 40 (89\%) reported symptomatic relief soon after FMT, with 18 patients reporting normal defecation during the follow up of 9 to 19 months. ${ }^{54}$ Although preliminary studies are promising, RCTs are needed to determine if FMT truly is an effective treatment modality for IBS or chronic constipation.

\section{Non-GI disorders}

FMT can also be used to treat diseases other than GI disorders in which the gut microbiota is disturbed. There are preliminary reports on the use of FMT therapy in a wide range of disorders including Parkinson's disease, fibromyalgia, chronic fatigue syndrome, myoclonus dystonia, multiple sclerosis, obesity, insulin resistance, metabolic syndrome, and childhood regressive autism (Table 1$){ }^{2}$ Vrieze et al. ${ }^{55}$ performed 
Table 1. Disorders Associated with an Altered Intestinal Microbiota

\begin{tabular}{|c|c|}
\hline Gastrointestinal & Non-gastrointestinal \\
\hline Cholelithiasis & Arthritis \\
\hline Colorectal cancer & Asthma \\
\hline Hepatic encephalopathy & Atopy \\
\hline Idiopathic constipation $^{\text {a) }}$ & Autism ${ }^{\text {a) }}$ \\
\hline Inflammatory bowel disease ${ }^{\text {b) }}$ & Autoimmune disorder \\
\hline Irritable bowel syndrome ${ }^{a}$ & Chronic fatigue syndrome ${ }^{a}$ \\
\hline Familial Mediterranean fever & Diabetes mellitus and insulin resistance $\mathrm{e}^{\mathrm{b})}$ \\
\hline Gastric carcinoma and lymphoma & Eczema \\
\hline \multirow[t]{13}{*}{ Recurrent Clostridium difficile infection ${ }^{\text {b) }}$} & Fibromyalgia $^{\text {a) }}$ \\
\hline & Hay fever \\
\hline & Hypercholesterolemia \\
\hline & Idiopathic thrombocytopenic purpura ${ }^{\text {a) }}$ \\
\hline & Ischemic heart disease \\
\hline & Metabolic syndrome ${ }^{a}$ \\
\hline & Mood disorders \\
\hline & Multiple sclerosis ${ }^{\mathrm{a})}$ \\
\hline & Myoclonus dystonia $^{\text {a) }}$ \\
\hline & Nonalcoholic fatty liver disease \\
\hline & Obesity \\
\hline & Oxalic acid kidney stones \\
\hline & Parkinson's disease $\mathrm{e}^{\mathrm{a})}$ \\
\hline
\end{tabular}

${ }^{a}$ Beneficial effect fecal microbiota transplantation (FMT) in case series; ${ }^{\text {b) }}$ Beneficial effect FMT in randomized clinical trials.

RCT of FMT in 18 male patients with metabolic syndrome. Patients who received fecal microbiota infusion from lean male donors reported a marked increase in insulin sensitivity and levels of butyrate-producing intestinal microbiota after 6-week infusion, whereas no significant changes were seen in the control group.

\section{FMT PROCEDURES}

FMT carries the possibility of transmitting infectious agents, and therefore, rigorous screening tests are recommended to reduce such risk (Fig. 1). ${ }^{56}$ Once a donor is selected, blood and fecal samples must be tested for pathogens. Current guidelines by an FMT workgroup in the United States ${ }^{57}$ recommend using a donor questionnaire that is similar to current protocols for screening blood donors. Donor exclusion criteria are shown in the "history" part of Fig. 1. The donors may be chosen from family members, intimate partners, friends, or unrelated volunteer. Intimate partners have the advantage of shared environmental risk factors, which may minimize the risk of infection transmission. ${ }^{57}$ In addition, maternal-line first-degree relatives may have the advantage of sharing the greatest number of microbial species in their intestinal microbiota with the recipient; thus, adaptive immune elements in the mucosal immune system (e.g., antigen-specific antibody) might be more tolerant of the microbiota from such donors. $^{57}$ The first systematic review of 317 patients with recurrent CDI reported that FMT from a related donor showed a slightly higher resolution rate (93\%) compared with unrelated donor (84\%). ${ }^{24}$ In addition, the difference in sex between donor and recipient had little impact on disease remission. However, these findings are still lacking evidence because recent meta-analysis did not show this difference. ${ }^{58}$ Unrelated volunteer donors may have an advantage when FMT is used to treat diseases in which genetics play a contributing factor, such as IBD. ${ }^{59}$ With regard to the preoperative preparation, recipients usually take large-volume bowel preparation regardless of the routes of administration. In some protocols, GI motility inhibitors such as loperamide are given to optimize retention of fecal microbiota contents. ${ }^{19}$ In addition, proton pump inhibitors should be administered to recipients who undergo FMT via upper GI route. The ideal fecal amount to be used for FMT has not been standardized. Practitioners 


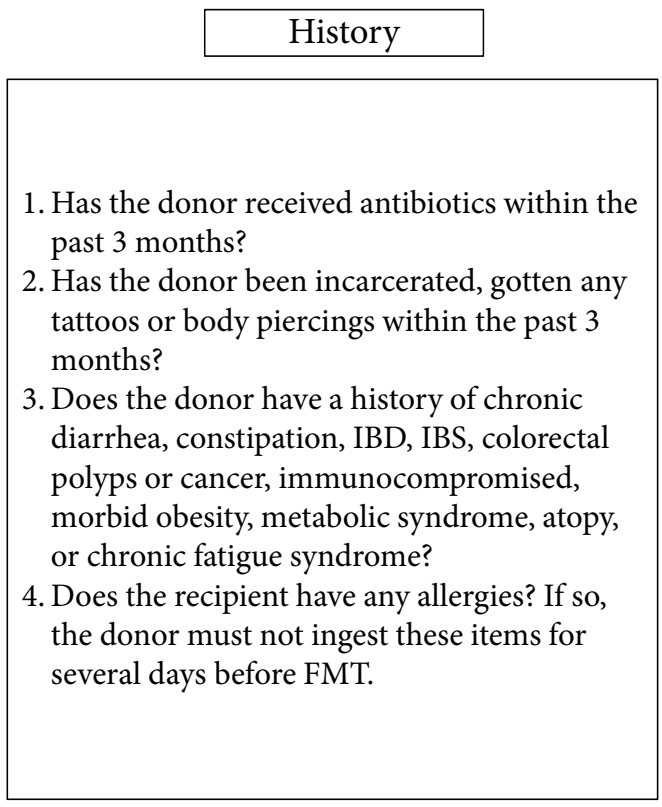

\begin{tabular}{|c|}
\hline Donor stool testing \\
\hline $\begin{array}{l}\text { 1. Clostridium difficile toxin } \\
\text { 2. Stool culture } \\
\text { 3. Stool ova and parasites } \\
\text { 4. Giardia stool antigen } \\
\text { 5. Helicobacter pylori stool antigen } \\
\text { 6. Cryptosporidium antigen test } \\
\text { 7. Isospora (acid fast stain) } \\
\text { 8. Rotavirus }\end{array}$ \\
\hline Donor serologic testing \\
\hline $\begin{array}{l}\text { 1. Hepatitis A IgM } \\
\text { 2. Hepatitis B surface antigen } \\
\text { 3. Antibodies to hepatitis B surface antigen } \\
\text { 4. Hepatitis C antibody } \\
\text { 5. HIV type } 1 \text { and } 2 \text { antibody } \\
\text { 6. Syphilis }\end{array}$ \\
\hline
\end{tabular}

Fig. 1. Donor and recipient screening for fecal microbiota transplantation. IBD, inflammatory bowel disease; IBS, irritable bowel syndrome; IgM, immunoglobulin M; FMT, fecal microbiota transplantation; HIV, human immunodeficiency virus.
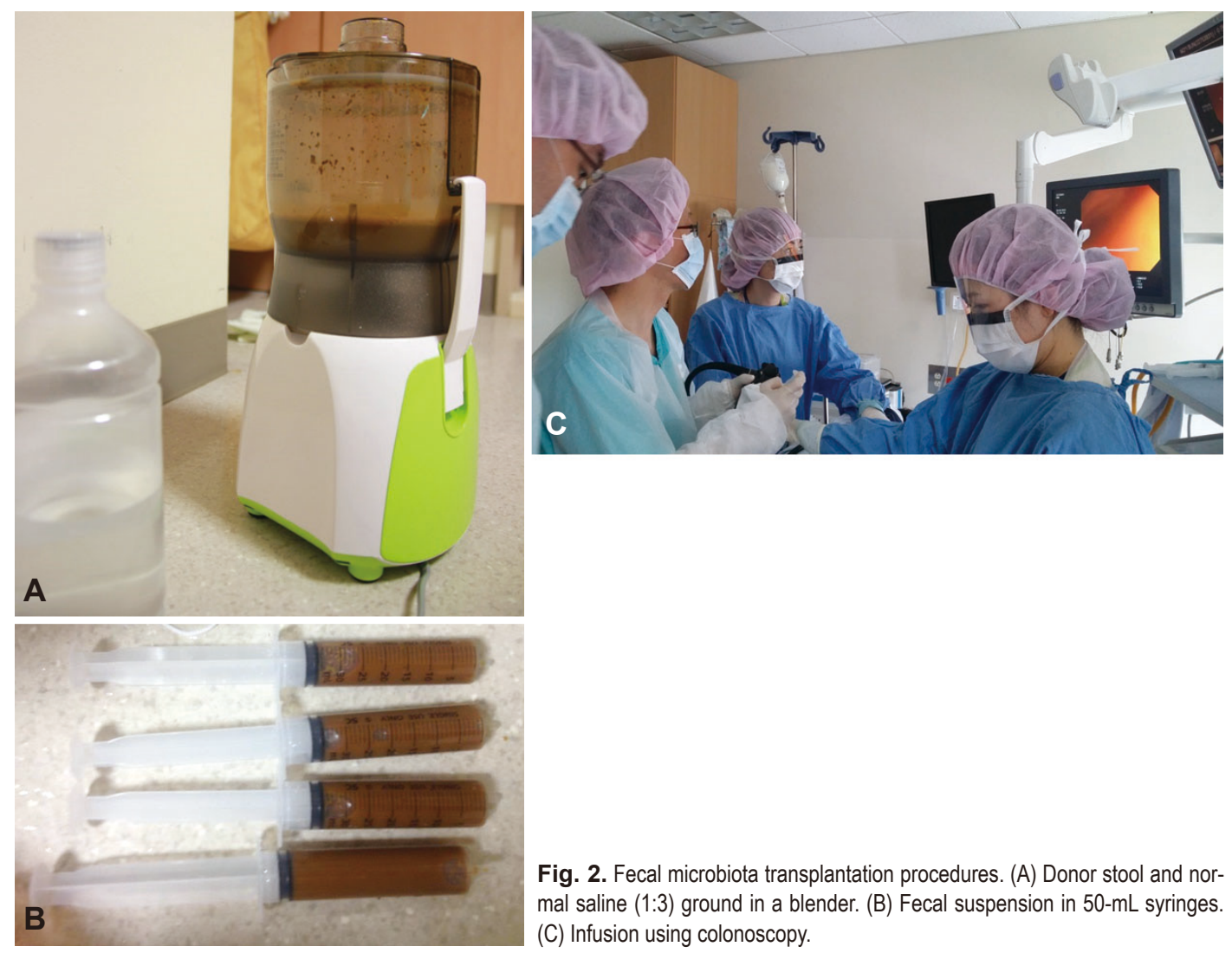

Fig. 2. Fecal microbiota transplantation procedures. (A) Donor stool and normal saline (1:3) ground in a blender. (B) Fecal suspension in 50-mL syringes. (C) Infusion using colonoscopy.

who regularly perform FMT favor 50 to $60 \mathrm{~g}$ of 250 to 300 $\mathrm{mL}$ diluent, respectively. ${ }^{56}$ Fig. 2 shows FMT procedures. A specimen of stool is suspended in tap or bottled water, milk, or nonbacteriostatic saline solution, although the latter is presumed to be less likely to affect the microbiota of donor stool. ${ }^{59}$ Donor stool is then homogenized either by hand stirring and shaking or using a mechanical blender. After suspension with the diluent, the mixture is filtered through a 
Table 2. Short-Term or Potential Long-Term Adverse Events of Fecal Microbiota Transplantation

\begin{tabular}{lcc}
\hline \multicolumn{1}{c}{ Short-term adverse events } & \multicolumn{1}{c}{ Serious events } & Potential long-term adverse events \\
\cline { 1 - 2 } Abdominal discomfort & $\begin{array}{c}\text { Complications of endoscopy (perforation, } \\
\text { bleeding) } \\
\text { Adverse effects related to sedation } \\
\text { (aspiration) }\end{array}$ & $\begin{array}{c}\text { Transmission of unrecognized infectious agents that cause } \\
\text { illness years later (e.g., hepatitis C, HIV) } \\
\text { Induction of chronic diseases based on alterations in the gut } \\
\text { microbiota (e.g., obesity, diabetes, atherosclerosis, IBD, colon } \\
\text { cancer, nonalcoholic fatty liver disease, IBS, asthma, autism) }\end{array}$ \\
Flatulence & $\begin{array}{c}\text { Transmission of enteric pathogens } \\
\text { Diarrhea }\end{array}$ & $\begin{array}{l}\text { Peritonitis in a patient undergoing } \\
\text { peritoneal dialysis }\end{array}$ \\
Constipation & Pneumonia & \\
Borborygmus & IBD flares \\
Vomiting & \\
Transient fever &
\end{tabular}

HIV, human immunodeficiency virus; IBD, inflammatory bowel disease; IBS, irritable bowel syndrome.

gauze or coffee filter or strained through a steel strainer to remove larger particulates. ${ }^{56}$ Administration of donor feces can be performed via the lower GI route, including colonoscopy, flexible sigmoidoscopy, rectal tube, or retention enema and/ or via the upper GI route such as nasogastric/nasointestinal tubes or gastroduodenoscopy. There is no definitive evidence for choosing any one modality over the other. Colonoscopic FMT is usually safe, well-tolerated, easily performed, and has the advantage of allowing examination of the entire colon; however, it must be selected carefully in patients with severe colitis and significant colonic distention due to a higher risk of perforation. ${ }^{19}$ In such cases, retention enema and flexible sigmoidoscopy could be alternative options, but they may be difficult for some patients to retain the transplanted stool and repeated, small-volume infusions over the course of 2 to 3 days may be required. FMT via the upper GI route is easy to perform and has a low risk. However, it may be uncomfortable and have some risk of vomiting and aspiration. ${ }^{59}$ In addition, it has some problems that donor stool may not be distributed throughout the entire colon and increase the risk of small intestinal bacterial overgrowth. Further studies are required to evaluate standardized and optimal route for FMT according to clinical situations.

\section{POTENTIAL ADVERSE EVENTS}

FMT may be safe and well tolerated with few serious adverse events, even though it is often administered to patients with significant medical comorbid conditions. ${ }^{59}$ Table 2 shows shortterm or potential long-term adverse events. Commonly reported immediate adverse events after FMT include abdominal discomfort, bloating, flatulence, diarrhea, constipation, vomiting, and transient fever. ${ }^{19,20}$ Most of these symptoms are self-limiting and disappear within 2 days after FMT. However, very little information is available regarding the long-term immunologic effects of FMT, including the onset of latent infections. In addition, diseases or conditions related to changes in gut microbiota may occur, including obesity, diabetes, atherosclerosis, IBD, colon cancer, nonalcoholic fatty liver disease, IBS, asthma, and autism. Long-term follow-up data for FMT should be investigated further. ${ }^{59}$ In a recent systematic review of FMT for recurrent CDI, no severe adverse events were reported. ${ }^{8}$ However, several safety concerns have been raised. A further concern is that the fame of FMT may lead to patients to use a "do-it-yourself" approach, without medical control, with possibly harmful consequences. ${ }^{60}$ De Leon et al. ${ }^{61}$ reported a UC patient who had been quiescent for more than 20 years and developed a flare of UC after FMT. This case report cautions us in utilizing FMT to treat CDI with UC. Moreover, a recent paper reported a UC patient who suffered cytomegalovirus infection after performing FMT without donor screening. ${ }^{62}$ As extracts of feces are mediators between the donor and the recipient, FMT has the potential for transmitting occult infections even when strict donor screening is performed. Possible transmission of norovirus infection through colonoscopic FMT has been reported but has not been proven definitely; ${ }^{63}$ it was hypothesized that the infection could have, in one case, originated from a medical personnel participating in the procedure. Future attempts to treat $\mathrm{CDI}$ or alter the microbiota of patients with IBD for treatment of IBD alone should be done with caution. Prospective controlled trials of FMT in CDI and other GI diseases to evaluate safety and efficacy will help further delineate indications, risks, and benefits. 


\section{CONCLUSIONS}

The high success rate and safety in the short-term reported for recurrent CDI has elevated FMT as an emerging treatment modality for a wide spectrum of other conditions associated with intestinal dysbiosis. Although the role of FMT in primary and severe CDI has not been established, FMT is recommended in case of two or more recurrences of uncomplicated CDI ${ }^{64}$ However, there remain many unanswered questions regarding FMT. The US Food and Drug Administration have determined that fecal microbiota products collected from healthy individuals is a biological product and a drug, requiring an investigational new drug application. Therefore, large RCTs to support safety and efficacy are necessary. In addition to regulatory issues, various issues to be considered include donor selection and screening, standardized protocols of stool preparation and route of administration, number of infusion and quantity of infused material, recipient preparation, and long-term safety. Furthermore, microbial restoration mechanisms should be defined with recent technological advances, including improvements in sequencing and computational biology. Finally, there is a need to develop new delivery methods to improve the accessibility. In the future, convenient equipment for stool preparation and oral formulations filled with fecal suspension or freeze-dried preparations will provide less aesthetic concerns, greater convenience, and maybe higher efficacy.

\section{Conflicts of Interest}

The authors have no financial conflicts of interest.

\section{REFERENCES}

1. Hooper LV, Littman DR, Macpherson AJ. Interactions between the microbiota and the immune system. Science 2012;336:1268-1273.

2. Xu MQ, Cao HL, Wang WQ, et al. Fecal microbiota transplantation broadening its application beyond intestinal disorders. World J Gastroenterol 2015;21:102-111.

3. Smits LP, Bouter KE, de Vos WM, Borody TJ, Nieuwdorp M. Therapeutic potential of fecal microbiota transplantation. Gastroenterology 2013;145:946-953.

4. Borody TJ, Warren EF, Leis S, Surace R, Ashman O. Treatment of ulcerative colitis using fecal bacteriotherapy. J Clin Gastroenterol 2003;37:4247.

5. Zhang F, Luo W, Shi Y, Fan Z, Ji G. Should we standardize the 1,700-year-old fecal microbiota transplantation? Am J Gastroenterol 2012;107:1755.

6. Brandt LJ, Aroniadis OC, Mellow M, et al. Long-term follow-up of colonoscopic fecal microbiota transplant for recurrent Clostridium difficile infection. Am J Gastroenterol 2012;107:1079-1087.

7. Eiseman B, Silen W, Bascom GS, Kauvar AJ. Fecal enema as an adjunct in the treatment of pseudomembranous enterocolitis. Surgery 1958;44:854-859.

8. Drekonja D, Reich J, Gezahegn S, et al. Fecal microbiota transplantation for clostridium difficile infection: a systematic review. Ann Intern Med
2015;162:630-638.

9. Human Microbiome Project Consortium. Structure, function and diversity of the healthy human microbiome. Nature 2012;486:207-214.

10. Qin J, Li R, Raes J, et al. A human gut microbial gene catalogue established by metagenomic sequencing. Nature 2010;464:59-65.

11. Sommer F, Bäckhed F. The gut microbiota: masters of host development and physiology. Nat Rev Microbiol 2013;11:227-238.

12. Britton RA, Young VB. Role of the intestinal microbiota in resistance to colonization by Clostridium difficile. Gastroenterology 2014;146:15471553.

13. Sullivan A, Edlund C, Nord CE. Effect of antimicrobial agents on the ecological balance of human microflora. Lancet Infect Dis 2001;1:101114.

14. Khoruts A, Dicksved J, Jansson JK, Sadowsky MJ. Changes in the composition of the human fecal microbiome after bacteriotherapy for recurrent Clostridium difficile-associated diarrhea. J Clin Gastroenterol 2010;44:354-360.

15. Borgia G, Maraolo AE, Foggia M, Buonomo AR, Gentile I. Fecal microbiota transplantation for Clostridium difficile infection: back to the future. Expert Opin Biol Ther 2015;15:1001-1014.

16. Cammarota G, Ianiro G, Cianci R, Bibbò S, Gasbarrini A, Currò D. The involvement of gut microbiota in inflammatory bowel disease pathogenesis: potential for therapy. Pharmacol Ther 2015;149:191-212.

17. Frank DN, St Amand AL, Feldman RA, Boedeker EC, Harpaz N, Pace NR. Molecular-phylogenetic characterization of microbial community imbalances in human inflammatory bowel diseases. Proc Natl Acad Sci U S A 2007;104:13780-13785.

18. Manichanh C, Borruel N, Casellas F, Guarner F. The gut microbiota in IBD. Nat Rev Gastroenterol Hepatol 2012;9:599-608.

19. Wang ZK, Yang YS, Chen Y, Yuan J, Sun G, Peng LH. Intestinal microbiota pathogenesis and fecal microbiota transplantation for inflammatory bowel disease. World J Gastroenterol 2014;20:14805-14820.

20. Colman RJ, Rubin DT. Fecal microbiota transplantation as therapy for inflammatory bowel disease: a systematic review and meta-analysis. J Crohns Colitis 2014;8:1569-1581.

21. Bartlett JG, Chang TW, Gurwith M, Gorbach SL, Onderdonk AB. Antibiotic-associated pseudomembranous colitis due to toxin-producing clostridia. N Engl J Med 1978;298:531-534.

22. van Nood E, Vrieze A, Nieuwdorp M, et al. Duodenal infusion of donor feces for recurrent Clostridium difficile. N Engl J Med 2013;368:407-415.

23. Youngster I, Sauk J, Pindar C, et al. Fecal microbiota transplant for relapsing Clostridium difficile infection using a frozen inoculum from unrelated donors: a randomized, open-label, controlled pilot study. Clin Infect Dis 2014;58:1515-1522.

24. Gough E, Shaikh H, Manges AR. Systematic review of intestinal microbiota transplantation (fecal bacteriotherapy) for recurrent Clostridium difficile infection. Clin Infect Dis 2011;53:994-1002.

25. Debast SB, Bauer MP, Kuijper EJ; European Society of Clinical Microbiology and Infectious Diseases. European Society of Clinical Microbiology and Infectious Diseases: update of the treatment guidance document for Clostridium difficile infection. Clin Microbiol Infect 2014;20 Suppl 2:1-26.

26. Surawicz CM, Brandt LJ, Binion DG, et al. Guidelines for diagnosis, treatment, and prevention of Clostridium difficile infections. Am J Gastroenterol 2013;108:478-498.

27. Petrof EO, Gloor GB, Vanner SJ, et al. Stool substitute transplant therapy for the eradication of Clostridium difficile infection: 'RePOOPulating' the gut. Microbiome 2013;1:3.

28. Youngster I, Russell GH, Pindar C, Ziv-Baran T, Sauk J, Hohmann EL. Oral, capsulized, frozen fecal microbiota transplantation for relapsing Clostridium difficile infection. JAMA 2014;312:1772-1778.

29. Satokari R, Mattila E, Kainulainen V, Arkkila PE. Simple faecal preparation and efficacy of frozen inoculum in faecal microbiota transplantation for recurrent Clostridium difficile infection: an observational cohort study. Aliment Pharmacol Ther 2015;41:46-53. 
30. Patel NC, Griesbach CL, DiBaise JK, Orenstein R. Fecal microbiota transplant for recurrent Clostridium difficile infection: Mayo Clinic in Arizona experience. Mayo Clin Proc 2013;88:799-805.

31. Hamilton MJ, Weingarden AR, Sadowsky MJ, Khoruts A. Standardized frozen preparation for transplantation of fecal microbiota for recurrent Clostridium difficile infection. Am J Gastroenterol 2012;107:761-767.

32. Gweon TG, Choi MG, Lee SK, et al. Two cases of refractory pseudomembranous colitis that healed following fecal microbiota transplantation. Korean J Med 2013;84:395-399.

33. Kim JE, Gweon TG, Yeo CD, et al. A case of Clostridium difficile infection complicated by acute respiratory distress syndrome treated with fecal microbiota transplantation. World J Gastroenterol 2014;20:1268712690.

34. Gweon TG, Lee KJ, Kang DH, et al. A case of toxic megacolon caused by clostridium difficile infection and treated with fecal microbiota transplantation. Gut Liver 2015;9:247-250.

35. Jang MO, An JH, Jung SI, Park KH. Refractory Clostridium difficile infection cured with fecal microbiota transplantation in vancomycin-resistant enterococcus colonized patient. Intest Res 2015;13:80-84.

36. Bennet JD, Brinkman M. Treatment of ulcerative colitis by implantation of normal colonic flora. Lancet 1989;1:164.

37. Borody TJ, George L, Andrews P, et al. Bowel-flora alteration: a potential cure for inflammatory bowel disease and irritable bowel syndrome? Med J Aust 1989;150:604.

38. Borody T, Wettstein A, Campbell J, et al. Fecal microbiota transplantation in ulcerative colitis: review of 24 years experience. Am J Gastroenterol 2012;107(Suppl 1):S665.

39. Vermeire S, Joossens M, Verbeke K, et al. Pilot study on the safety and efficacy of faecal microbiota transplantation in refractory crohn's disease. Gastroenterology 2012;142(5 Suppl 1):S360.

40. Anderson JL, Edney RJ, Whelan K. Systematic review: faecal microbiota transplantation in the management of inflammatory bowel disease. Aliment Pharmacol Ther 2012;36:503-516.

41. Sha S, Liang J, Chen M, et al. Systematic review: faecal microbiota transplantation therapy for digestive and nondigestive disorders in adults and children. Aliment Pharmacol Ther 2014;39:1003-1032.

42. Moayyedi P, Surette MG, Kim PT, et al. Fecal microbiota transplantation induces remission in patients with active ulcerative colitis in a randomized controlled trial. Gastroenterology 2015;149:102-109.

43. Rossen NG, Fuentes S, van der Spek MJ, et al. Findings from a randomized controlled trial of fecal transplantation for patients with ulcerative colitis. Gastroenterology 2015;149:110-118.

44. Borody TJ, Finlayson S, Paramsothy S. Is Crohn's disease ready for fecal microbiota transplantation? J Clin Gastroenterol 2014;48:582-583.

45. Zhang FM, Wang HG, Wang M, Cui BT, Fan ZN, Ji GZ. Fecal microbiota transplantation for severe enterocolonic fistulizing Crohn's disease. World J Gastroenterol 2013;19:7213-7216.

46. Gordon H, Harbord M. A patient with severe Crohn's colitis responds to faecal microbiota transplantation. J Crohns Colitis 2014;8:256-257.

47. Kao D, Hotte N, Gillevet P, Madsen K. Fecal microbiota transplantation inducing remission in Crohn's colitis and the associated changes in fecal microbial profile. J Clin Gastroenterol 2014;48:625-658.

48. Cui B, Feng Q, Wang H, et al. Fecal microbiota transplantation through mid-gut for refractory Crohn's disease: safety, feasibility, and efficacy trial results. J Gastroenterol Hepatol 2015;30:51-58.

49. Simrén M, Barbara G, Flint HJ, et al. Intestinal microbiota in functional bowel disorders: a Rome foundation report. Gut 2013;62:159-176.

50. Shanahan F, Quigley EM. Manipulation of the microbiota for treatment of IBS and IBD-challenges and controversies. Gastroenterology 2014;146:1554-1563.

51. Pinn DM, Aroniadis OC, Brandt LJ. Is fecal microbiota transplantation (FMT) an effective treatment for patients with functional gastrointestinal disorders (FGID)? Neurogastroenterol Motil 2015;27:19-29.

52. Rajilić-Stojanović M, Biagi E, Heilig HG, et al. Global and deep molecular analysis of microbiota signatures in fecal samples from patients with irritable bowel syndrome. Gastroenterology 2011;141:1792-1801.

53. Pinn DM, Aroniadis OC, Brandt LJ. Is fecal microbiota transplantation the answer for irritable bowel syndrome? A single-center experience. Am J Gastroenterol 2014;109:1831-1832.

54. Andrews P, Borody TJ, Shortis NP, Thompson S. Bacteriotherapy for chronic constipation: a long term follow-up. Gastroenterology 1995;108(4 Suppl 2):A563.

55. Vrieze A, Van Nood E, Holleman F, et al. Transfer of intestinal microbiota from lean donors increases insulin sensitivity in individuals with metabolic syndrome. Gastroenterology 2012;143:913-916.e7.

56. Brandt LJ, Aroniadis OC. An overview of fecal microbiota transplantation: techniques, indications, and outcomes. Gastrointest Endosc 2013;78:240-249.

57. Bakken JS, Borody T, Brandt LJ, et al. Treating Clostridium difficile infection with fecal microbiota transplantation. Clin Gastroenterol Hepatol 2011;9:1044-1049.

58. Kassam Z, Lee CH, Yuan Y, Hunt RH. Fecal microbiota transplantation for Clostridium difficile infection: systematic review and meta-analysis. Am J Gastroenterol 2013;108:500-508.

59. Kelly CR, Kahn S, Kashyap P, et al. Update on fecal microbiota transplantation 2015: indications, methodologies, mechanisms, and outlook. Gastroenterology 2015;149:223-237.

60. El-Matary W. Fecal microbiota transplantation: long-term safety issues. Am J Gastroenterol 2013;108:1537-1538.

61. De Leon LM, Watson JB, Kelly CR. Transient flare of ulcerative colitis after fecal microbiota transplantation for recurrent Clostridium difficile infection. Clin Gastroenterol Hepatol 2013;11:1036-1038.

62. Hohmann EL, Ananthakrishnan AN, Deshpande V. Case records of the Massachusetts General Hospital. Case 25-2014. A 37-year-old man with ulcerative colitis and bloody diarrhea. N Engl J Med 2014;371:668-675.

63. Schwartz M, Gluck M, Koon S. Norovirus gastroenteritis after fecal microbiota transplantation for treatment of Clostridium difficile infection despite asymptomatic donors and lack of sick contacts. Am J Gastroenterol 2013;108:1367.

64. Bagdasarian N, Rao K, Malani PN. Diagnosis and treatment of Clostridium difficile in adults: a systematic review. JAMA 2015;313:398-408. 